\title{
Use of q-values to improve a genetic algorithm to identify robust gene signatures
}

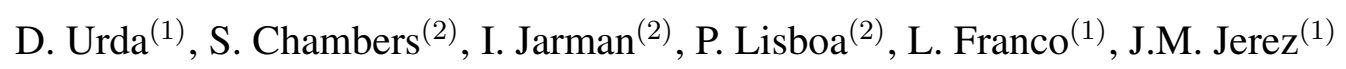

(1) Department of Computer Science, University of Málaga

Bulevar Louis Pasteur, 35, 29071 Málaga, Spain, \{durda,lfranco,jja\} @lcc.uma.es

(2) School of Computing and Mathematical Sciences, Liverpool John Moores University

Byrom Street, Liverpool L3 3AF, United Kingdom, \{s.j.chambers,i.h.jarman,p.j.lisboa\}@ljmu.ac.uk

Keywords: DNA microarrays, Evolutionary algorithms, t-test, q-values, Feature selection.

Abstract. Several approaches have been proposed for the analysis of DNA microarray datasets, focusing on the performance and robustness of the final feature subsets. The novelty of this paper arises in the use of q-values to pre-filter the features of a DNA microarray dataset identifying the most significant ones and including this information into a genetic algorithm for further feature selection. This method is applied to a lung cancer microarray dataset resulting in similar performance rates and greater robustness in terms of selected features (on average a $36.21 \%$ of robustness improvement) when compared to results of the standard algorithm.

\section{Scientific Background}

DNA microarray technology has been widely used for gene expression profiling and prediction of cancer. Analysis of such data involves facing a problem commonly referred to as the curse of dimensionality [9] where each sample is described by thousands of features (genes) with few samples - often fewer than a hundred - available. Several approaches have been proposed to identify relevant genes with good performance in classifying the disorder under investigation. However, these approaches lack a desirable feature when identifying gene expression profiles - robustness. A common feature of such methods is instability of results with high variability of identified features when repeated executions of the algorithm are made. To tackle this problem, recent works have proposed different methodologies that try to achieve robust feature subset selections with good performance rates in test data $[7,10]$.

Use of statistical tests with multiple features against some null hypothesis is common practice with the expectation that a proportion of such features would be incorrectly considered significant [8]. In such circumstances it is important to use some form of false discovery rate technique to either adjust the p-values [1] or use a different measure which takes into account false positives such as the q-value [8]. Use of such a measure allows focus to be placed on features which can be considered to satisfy a null hypothesis in further analysis. In the original paper [8] this methodology reduced the number of features identified in the Hedenfalk dataset from 605 to 162 within a total feature set of 3170 .

In this paper a modified t-test and q-values [8] are incorporated into a feature selection procedure similar to the genetic algorithm (GA) described in [7] with the purpose of identifying genes that are significant in differentiating lung cancer microarray expressions. In their approach, biological information from KEGG [5, 6] database was included into the GA resulting in more robust feature subsets with good performance 
rates. The expectation of introducing a subset of genes, selected using q-values, into the GA would be for better and more robust solutions than the original results from the GA.

The rest of the paper is structured as follows: Section 2 describes the dataset used in this study as well as the methodology applied; Section 3 shows the results obtained in this work and a comparison to previous results of one similar approach; and Section 4 provides some conclusions.

\section{Materials and Methods}

A freely available ${ }^{1}$ high dimensional biomedical dataset has been used throughout this work, comprising 181 tissue samples of two types of lung cancer, malignant pleural Mesothelioma (MPM) and Adenocarcinoma (ADCA) [4]. Samples are unbalanced with 31 corresponding to MPM and 150 ADCA, described in each case by 12533 genes. The Affymetrix ID for the lung cancer DNA microarray dataset is hgu95a and the R package "hgu95a.db" [2] was used to manage and pre-process biological information related to this microarray. For the analysis, the dataset was separated into training and test sets, comprising 80 samples and 101 samples respectively with care taken to keep the same proportion of both MPM and ADCA classes.

The novelty of this approach is the introduction of a more robust statistical method with the expectation of an improvement in the robustness of the final obtained subset of features with direct biological relevance, evidenced by the maintaining of good generalisation in the validation results.

\subsection{Significance Testing}

A permutation based modified t-test [8] was used to evaluate the null hypothesis that there is no difference in expression between the two different groups (MDM and ADCA) accounting for the different variance within each group. The two sample t-statistic for a given gene is expressed as in (1), and the p-values estimated as per (2). In this case $\overline{x_{1}}$ and $\overline{x_{2}}$ represent the means of group 1 and group 2, with $s_{1}^{2}$ and $s_{2}^{2}$ being the respective variances. $B$ is the number of re-samples taken for the modified t-test (a value of $\mathrm{B}=100$ was used), $n$ the number of features and $t$ the value for a given t-statistic ( $t_{1}^{0 b}$ to $t_{n}^{0 b}$ are the set of null statistics calculated using the resampling procedure).

$$
\begin{gathered}
t=\frac{\bar{x}_{1}-\bar{x}_{2}}{\sqrt{\frac{s_{1}^{2}}{n_{1}}+\frac{s_{2}^{2}}{n_{2}}}} \\
p_{i}=\sum_{b=1}^{B} \frac{\#\left\{j:\left|t_{j}^{0 b}\right| \geq\left|t_{i}\right|, j=1, \ldots, n\right\}}{n \times B}
\end{gathered}
$$

This approach has as the null hypothesis that there is no difference in expression between the two genes and that the t-statistic holds to the same distribution across both [8].

Using the p-values from this study, an FDR-based significance measure, q-values [8], was used to select only those genes significant at the 5\% level for inclusion in the model estimation stage. These q-values are an important tool in determining significance of features, and particularly so in genome studies, as they implicitly account for multiple testing, and allow for a more accurate determination of the expected false-positives from the inclusion of a particular feature.

\subsection{Model estimation}

In this paper, the strategy proposed in [7] is used with some slight changes. The strategy consists of two separate stages:

\footnotetext{
${ }^{1}$ http://cilab.ujn.edu.cn/datasets.htm
} 
- The 228 pathways identified as related to lung cancer disease are analysed to produce a ranking which allows selection of the best pathways. In contrast to the first stage in [7] only the training dataset of 80 samples is analysed by applying a 10-fold cross-validation strategy. The purpose at this stage is to obtain an accuracy measure and identify the number of keywords for each pathway using a text-mining procedure, following the same process as [7] but refined using genes identified as significant in Section 2.1.

- Using pathways identified from the first stage as being of importance, a genetic algorithm is applied using the fitness function from (3) where $\lambda, \beta \in[0,1)$ and $\lambda+\beta<1, k$ is the number of selected features, 100 is a normalization factor due to the limited number of active features in a chromosome, and function score $(\mathbf{x})$ which estimates the biological relevance of the selected features. This function has been modified to (4) where $M$ and $N$ are normalization factors representing the number of significant genes on the pathway and total number of significant genes on all pathways respectively. $i$ is the number of selected significant genes included in the pathway being analysed and $j$ the number of selected significant genes not in the pathway such that $i+j=k$. To obtain the accuracy rate for (3), Linear Discriminant Analysis (LDA) [3] is used by applying 10-k-fold cross validation to each chromosome analysed within the GA execution.

- The final step validates the performance of the selected model by performing LDA on the training data and applying the results to the larger test dataset to obtain the prediction accuracy. LDA was chosen in order to make a fair comparison to results previously published in [7] as well as for its simplicity.

$$
\begin{gathered}
\operatorname{fitness}(\mathbf{x})=(1-\lambda-\beta)(1-A C C(\mathbf{x}))+\lambda \frac{k}{100}+\beta \operatorname{score}(\mathbf{x}), \\
\operatorname{score}(\mathbf{x})=\frac{\left(1-\frac{i}{M}\right)+\left(1-\frac{j}{N-M}\right)}{2},
\end{gathered}
$$

\section{Results}

The predictive capability and the number of relevant keywords were calculated for each of the 228 pathways. Table 1 details the best pathways according to Accuracy (Acc) values using the genes identified as being significant at $q<0.05$ as the first sorting criterion, and the number of keywords found during the text mining of the pathway descriptions in the KEGG database as the second criterion. Bold rows correspond to pathways which ranked in the top 10 found in [7] during the first stage. Those in bold-italic are the six best pathways selected to be analysed on the second stage of the methodology previously. Of note is that the top 10 pathways from the original work are ranked in the overall top 27 pathways ( $<12 \%$ of total), and pathway " 04610 " being the only one exhibiting minimal decline in ranking.

Instead of selecting the best pathways using this ranking as previously done, for comparative purposes the six best pathways from the previous work were selected [7] for analysis using the modified GA presented in Section 2.2 using the test/train datasets for model estimation and validation. This stage of the analysis was repeated 100 times for each of the six pathways to obtain estimates of the model accuracy.

Table 2 shows on average a perfomance comparison using the GA approach published in [7] and our proposal in this paper. In terms of prediction accuracy, both approaches obtain similar performance (approximately 95\% depending on the analysed pathway). However, the main advantage of the present approach arises while analyzing the robustness of the subset of features selected. This robustness measure is obtained by 
Table 1: Pathways ranked by prediction ability using significant genes and number of keywords found. Bold rows correspond to pathways which ranked in the top 10 according to [7] (See the text for more details).

\begin{tabular}{|c|c|c|c|c|c|c|c|}
\hline Rank & Code & Pathway & \#Genes & $A c c$ & \#Genes 0.05 & Acc 0.05 & \#Keywords \\
\hline 1 & 04020 & Calcium signaling pathway & 246 & 0.933 & 28 & 0.9975 & $0 / 1116$ \\
\hline 2 & 04144 & Endocytosis & 244 & 0.99 & 32 & 0.99 & $0 / 506$ \\
\hline 3 & 04650 & $\begin{array}{l}\text { Natural killer cell mediated } \\
\text { cytotoxicity }\end{array}$ & 172 & 0.915 & 13 & 0.9875 & $3 / 871$ \\
\hline 4 & 04010 & MAPK signaling pathway & 423 & 0.935 & 37 & 0.9875 & $2 / 609$ \\
\hline 5 & 04062 & Chemokine signaling pathway & 254 & 0.945 & 29 & 0.9875 & $1 / 901$ \\
\hline 6 & 04141 & $\begin{array}{l}\text { Protein processing in endoplasmic } \\
\text { reticulum }\end{array}$ & 181 & 0.908 & 14 & 0.9875 & $1 / 458$ \\
\hline 7 & 01100 & Metabolic pathways & 970 & 0.975 & 146 & 0.9875 & $0 / 116$ \\
\hline 8 & 00230 & Purine metabolism & 148 & 0.93 & 19 & 0.9875 & $0 / 271$ \\
\hline 9 & 04270 & $\begin{array}{c}\text { Vascular smooth muscle } \\
\text { contraction }\end{array}$ & 149 & 0.973 & 28 & 0.9875 & $0 / 891$ \\
\hline 10 & 00240 & Pyrimidine metabolism & 83 & 0.975 & 15 & 0.9875 & 0/150 \\
\hline 11 & 04510 & Focal adhesion & 320 & 0.955 & 42 & 0.985 & $1 / 824$ \\
\hline 12 & 05200 & Pathways in cancer & 557 & 0.96 & 63 & 0.9825 & $11 / 4504$ \\
\hline 20 & 04530 & Tight junction & 158 & 0.965 & 27 & 0.9775 & $1 / 545$ \\
\hline 25 & 04360 & Axon guidance & 166 & 0.975 & 22 & 0.975 & $0 / 427$ \\
\hline 27 & 04514 & Cell adhesion molecules (CAMs) & 154 & 0.95 & 25 & 0.975 & $0 / 921$ \\
\hline 45 & 04610 & $\begin{array}{c}\text { Complement and coagulation } \\
\text { cascades }\end{array}$ & 73 & 0.978 & 14 & 0.965 & $3 / 660$ \\
\hline
\end{tabular}

Table 2: Comparison of original approach and proposed approach. Columns 2-4 show the mean of each of No. of Genes, genes in pathway and significant genes in pathway with standard deviations. Additionally, column 5 shows the robustness of results and the last column the accuracy.

\begin{tabular}{|c|c|c|c|c|c|c|}
\hline & Pathway & \#Genes & \#Genes in pathway & \#Genes significant in pathway & Robustness & Accuracy \\
\hline 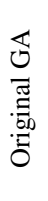 & $\begin{array}{l}04144 \\
04530 \\
04514 \\
04610 \\
04010 \\
05200\end{array}$ & $\begin{array}{l}4.43 \pm 1.00 \\
4.22 \pm 1.05 \\
3.96 \pm 1.07 \\
3.85 \pm 1.02 \\
4.04 \pm 1.29 \\
3.86 \pm 1.52\end{array}$ & $\begin{array}{l}2.87 \pm 1.22 \\
2.79 \pm 1.11 \\
2.32 \pm 1.29 \\
2.94 \pm 1.24 \\
1.71 \pm 1.09 \\
1.51 \pm 1.20\end{array}$ & $\begin{array}{l}1.75 \pm 1.19 \\
2.04 \pm 1.04 \\
1.49 \pm 0.95 \\
1.63 \pm 1.00 \\
0.88 \pm 0.83 \\
0.68 \pm 0.79\end{array}$ & $\begin{array}{c}0.1225 \\
0.14125 \\
0.135 \\
0.24455 \\
0.086667 \\
0.079\end{array}$ & $\begin{array}{c}0.9568 \pm 0.0229 \\
0.9630 \pm 0.0248 \\
0.9463 \pm 0.025 \\
0.9398 \pm 0.0197 \\
0.9445 \pm 0.0275 \\
0.9450 \pm 0.0249\end{array}$ \\
\hline 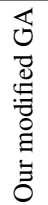 & $\begin{array}{l}04144 \\
04530 \\
04514 \\
04610 \\
04010 \\
05200\end{array}$ & $\begin{array}{l}4.82 \pm 1.31 \\
4.63 \pm 1.54 \\
5.59 \pm 1.55 \\
4.29 \pm 1.04 \\
3.54 \pm 1.14 \\
3.08 \pm 1.24\end{array}$ & $\begin{array}{l}3.96 \pm 1.54 \\
3.94 \pm 1.64 \\
4.94 \pm 1.79 \\
3.94 \pm 1.23 \\
2.58 \pm 1.33 \\
1.63 \pm 1.28\end{array}$ & $\begin{array}{l}3.66 \pm 1.63 \\
3.86 \pm 1.57 \\
4.89 \pm 1.76 \\
3.12 \pm 1.21 \\
2.28 \pm 1.32 \\
1.34 \pm 1.20\end{array}$ & $\begin{array}{c}0.148 \\
0.158 \\
0.21591 \\
0.29846 \\
0.15455 \\
0.098182\end{array}$ & $\begin{array}{c}0.9590 \pm 0.0201 \\
0.9732 \pm 0.0192 \\
0.9458 \pm 0.0261 \\
0.9439 \pm 0.02 \\
0.9431 \pm 0.0302 \\
0.932 \pm 0.0261\end{array}$ \\
\hline
\end{tabular}

averaging each gene frequency of appearance over the 100 GA executions, discarding those genes that do not appear more than a $5 \%$ of the times. In this sense, it should be highlighted that in two out of six pathways analysed, a $78.33 \%$ and $59.93 \%$ of improvement is reached in terms of robustness (pathways "04010" and "04514" respectively). Pathway " 04530 " is the one with lowest improvement (just $11.86 \%$ ), while for the remaining pathways the robustness was approximately increased by a $20 \%$.

The top eleven final selected features for each of the pathways as shown in Table 3 can be directly compared to those obtained in [7]. Consistency is apparent in these as at least four genes are present in the previous work (those highlighted in bold). Furthermore, because of the use of q-values to limit features to those which exhibit significant difference in expression, this approach yields results that contains a larger number of significant selected genes belonging to the top 11 features of a given pathway. These significant genes are also picked by the GA with greater frequency than those shown in the previous work, and thus the robustness of the present method should be higher, as 
indeed is as seen in Table 2 .

Table 3: Frequency of selection for the most frequently picked features in each pathway previously identified [7] as important. The notes column indicated by ${ }^{\dagger}$ highlights whether the gene is significant in pathway $(*)$, not significant but in pathway $(* *)$ or out of pathway $(* * *)$.

\begin{tabular}{l|l|l|c|l} 
ID & Symbol & Probe Set ID & Freq.(\%) & Note $^{\dagger}$ \\
\hline $\mathbf{2 5 2 0}$ & CLTB & 32522_f_at & 13.00 & $* *$ \\
1035 & KDR & 1954 at & 14.00 & $*$ \\
$\mathbf{6 3 3}$ & ERBB3 & $\mathbf{1 5 8 5}$ _at & $\mathbf{1 5 . 0 0}$ & $*$ \\
$\mathbf{1 1 8 2}$ & ERBB3 & 2089_s_at & $\mathbf{1 6 . 0 0}$ & $*$ \\
11052 & PARD3 & 40973 at & 16.00 & $*$ \\
$\mathbf{2 5 2 1}$ & CLTB & 32523_at & $\mathbf{2 2 . 0 0}$ & $*$ \\
12020 & DAB2 & $479 \_a t$ & 22.00 & $*$ \\
$\mathbf{9 7 5 8}$ & SH3GLB1 & 39691_at & $\mathbf{2 7 . 0 0}$ & $*$ \\
967 & NTRK1 & 1892_s_at & 28.00 & $*$ \\
3893 & RAB11FIP5 & 33882_at & 41.00 & $*$ \\
$\mathbf{9 8 6 3}$ & AP2M1 & 39795_at & $\mathbf{4 3 . 0 0}$ & $*$
\end{tabular}

\begin{tabular}{l|l|l|c|l} 
ID & Symbol & Probe Set ID & Freq. $(\%)$ & Note $^{\dagger}$ \\
\hline 6335 & PRKCZ & 362_at & 13.00 & $*$ \\
7453 & MYH11 & 37407_s_at & 14.00 & $*$ \\
12312 & MYH11 & 773 at & 14.00 & $*$ \\
3916 & CLDN3 & 33904_at & 15.00 & $*$ \\
$\mathbf{4 1 7 4}$ & ACTG1 & 34160_at & $\mathbf{1 9 . 0 0}$ & $*$ \\
$\mathbf{8 5 3 7}$ & CLDN7 & 38482_at & $\mathbf{2 0 . 0 0}$ & $*$ \\
$\mathbf{1 1 0 5 2}$ & PARD3 & 40973_at & $\mathbf{2 3 . 0 0}$ & $*$ \\
$\mathbf{8 3 9 3}$ & RRAS & 38338_at & $\mathbf{2 6 . 0 0}$ & $*$ \\
$\mathbf{2 0 3 9}$ & PRKCD & 32046_at & $\mathbf{3 2 . 0 0}$ & $*$ \\
3844 & SPTAN1 & 33833_at & 33.00 & $*$ \\
$\mathbf{5 3 0 1}$ & CLDN4 & 35276_at & $\mathbf{5 7 . 0 0}$ & $*$
\end{tabular}

(a) Lung Pathway 04144

\begin{tabular}{l|l|l|c|l} 
ID & Symbol & Probe Set ID & Freq. $(\%)$ & Note $^{\dagger}$ \\
\hline 1248 & PECAM1 & 268_at & 24.00 & $*$ \\
1718 & HLA-DOA & 31728_at & 25.00 & $*$ \\
$\mathbf{3 1 7 3}$ & NEO1 & 33169_at & $\mathbf{2 5 . 0 0}$ & $*$ \\
3916 & CLDN3 & 33904_at & 25.00 & $*$ \\
8509 & ICAM2 & 38454_g_at & 25.00 & $*$ \\
10372 & ICAM3 & $402 \_s \_a t$ & 27.00 & $*$ \\
$\mathbf{8 5 3 7}$ & CLDN7 & $\mathbf{3 8 4 8 2}$ at & $\mathbf{2 9 . 0 0}$ & $*$ \\
$\mathbf{1 1 4 3}$ & CDH2 & $\mathbf{2 0 5 3}$ at & $\mathbf{3 0 . 0 0}$ & $*$ \\
$\mathbf{8 5 0 8}$ & ICAM2 & 38453_at & $\mathbf{3 4 . 0 0}$ & $*$ \\
4217 & PVRL3 & 34202_at & 38.00 & $*$ \\
$\mathbf{5 3 0 1}$ & CLDN4 & 35276_at & $\mathbf{6 6 . 0 0}$ & $*$
\end{tabular}

(c) Lung Pathway 04514

\begin{tabular}{l|l|l|c|l} 
ID & Symbol & Probe Set ID & \multicolumn{1}{|c}{ Freq.(\%) } & Note $^{\dagger}$ \\
\hline 6700 & CD14 & 36661_s_at & 6.00 & $*$ \\
620 & PDGFB & 1573_at & 7.00 & $*$ \\
957 & MECOM & 1882 __at & 7.00 & $*$ \\
5104 & FGF9 & 35081_at & 9.00 & $*$ \\
$\mathbf{3 2 5 0}$ & MAPK13 & 33245_at & $\mathbf{1 1 . 0 0}$ & $*$ \\
5997 & HSPA6 & 35965_at & 14.00 & $*$ \\
909 & RRAS2 & $1838 \_$g_at & 16.00 & $*$ \\
$\mathbf{8 3 9 3}$ & RRAS & 38338_at & $\mathbf{1 6 . 0 0}$ & $*$ \\
$\mathbf{5 3 5 6}$ & FLNC & 35330_at & $\mathbf{1 7 . 0 0}$ & $*$ \\
967 & NTRK1 & 1892_s_at & 23.00 & $*$ \\
$\mathbf{6 6 7}$ & FGF9 & 1616_at & $\mathbf{4 4 . 0 0}$ & $*$
\end{tabular}

(e) Lung Pathway 04010

(b) Lung Pathway 04530

\begin{tabular}{c|l|l|l|l} 
ID & Symbol & Probe Set ID & Freq.(\%) & Note $^{\dagger}$ \\
\hline $\mathbf{6 5 8 1}$ & F3 & 36543_at & $\mathbf{1 1 . 0 0}$ & $*$ \\
5783 & PROS1 & 35752_s_at & 13.00 & $*$ \\
$\mathbf{6 8 2 1}$ & SERPINA1 & $\mathbf{3 6 7 8 1}$ at & $\mathbf{1 4 . 0 0}$ & $*$ \\
$\mathbf{8 4 9 6}$ & CD46 & 38441_s_at & $\mathbf{1 4 . 0 0}$ & $*$ \\
12146 & VWF & 607_s_at & 22.00 & $*$ \\
12211 & SERPINE1 & 672_at & 23.00 & $* *$ \\
$\mathbf{9 4 7 4}$ & C1R & 39409_at & $\mathbf{3 6 . 0 0}$ & $*$ \\
$\mathbf{5 7 2 7}$ & CFI & 35698_at & $\mathbf{4 5 . 0 0}$ & $*$ \\
$\mathbf{8 1 7 8}$ & SERPINE1 & 38125_at & $\mathbf{5 9 . 0 0}$ & $* *$ \\
$\mathbf{5 8 5 3}$ & CFB & 35822_at & $\mathbf{6 7 . 0 0}$ & $*$ \\
$\mathbf{9 8 4 3}$ & SERPING1 & 39775_at & $\mathbf{6 8 . 0 0}$ & $*$
\end{tabular}

(d) Lung Pathway 04610

\begin{tabular}{l|l|l|c|l} 
ID & Symbol & Probe Set ID & Freq.(\%) & Note $^{\dagger}$ \\
\hline 8813 & FADD & 38755_at & 6.00 & $*$ \\
12200 & GAS1 & 661 at & 6.00 & $* * *$ \\
6616 & BIRC2 & 36578_at & 7.00 & $*$ \\
411 & RARB & 1381 at & 8.00 & $*$ \\
7849 & SEMA3C & 377_g_at & 8.00 & $* * *$ \\
$\mathbf{8 3 7 0}$ & ALDH1A2 & 38315_at & $\mathbf{9 . 0 0}$ & $* * *$ \\
$\mathbf{5 1 0 4}$ & FGF9 & 35081_at & $\mathbf{1 0 . 0 0}$ & $*$ \\
967 & NTRK1 & 1892_s_at & 11.00 & $*$ \\
$\mathbf{1 1 3 6}$ & JUP & 2047_s_at & $\mathbf{1 3 . 0 0}$ & $*$ \\
10532 & STAT5A & 40458_at & 14.00 & $*$ \\
$\mathbf{6 6 7}$ & FGF9 & 1616_at & $\mathbf{1 6 . 0 0}$ & $*$
\end{tabular}

(f) Lung Pathway 05200

\section{Conclusion}

In this work, a lung cancer disease microarray dataset has been analysed in order to obtain a subset of genes with good predictive performance by using a previously published genetic algorithm modified to include a significance test based on the use of q-values. It has been shown that the inclusion of this information into the GA, to identify those genes having a significant difference in expression, has yielded results that are similar in performance to the original method but exhibiting improved robustness in terms of the selected features with an improvement between $11.86 \%-78.33 \%$ (average $36.21 \%$ ). This higher robustness observed is achieved as the search in the GA is now guided to genes previously identified as significant without discarding the potential utility of other genes. Moreover, these results are consistent with the original ones since in the top 11 most selected genes, 4 to 6 genes were also included within the results in the original work. Further work should consider a deeper biological analysis of these results and also further investigation of the predictive abilities of pathways either alone or in combination with those genes identified as significant in this study. 


\section{Acknowledgments}

The authors acknowledge support through Grants TIN2010-16556 from MICINNSPAIN and P08-TIC-04026 (Junta de Andalucía), all of which include FEDER funds.

\section{References}

[1] Benjamini, Y., And Hochberg, Y. Controlling the False Discovery Rate: A Practical and Powerful Approach to Multiple Testing. J. R. Stat. Soc. Ser. B 57, 1 (1995), 289-300.

[2] Carlson, M. hgu95a.db: Affymetrix Human Genome U95 Set annotation data (chip hgu95a), 2011.

[3] Duda, R. O., Hart, P. E., And Stork, D. G. Pattern Classification, 2nd editio ed. Wiley, 2000.

[4] Gordon, G. J., Jensen, R. V., Hsiao, L.-L., Gullans, S. R., BlumenStOck, J. E., Ramaswamy, S., Richards, W. G., Sugarbaker, D. J., AND BUENO, R. Translation of microarray data into clinically relevant cancer diagnostic tests using gene expression ratios in lung cancer and mesothelioma. Cancer Res. 62, 17 (Sept. 2002), 4963-7.

[5] Kanehisa, M., And Goto, S. KEGG: kyoto encyclopedia of genes and genomes. Nucleic Acids Res. 28, 1 (Jan. 2000), 27-30.

[6] Kanehisa, M., Goto, S., Sato, Y., Kawashima, M., Furumichi, M., And TANABE, M. Data, information, knowledge and principle: back to metabolism in KEGG. Nucleic Acids Res. 42, Database issue (Jan. 2014), D199-205.

[7] Luque-Baena, R. M., Urda, D., Gonzalo Claros, M., Franco, L., And JEREZ, J. M. Robust gene signatures from microarray data using genetic algorithms enriched with biological pathway keywords. J. Biomed. Inform. (Jan. 2014).

[8] Storey, J. D., and Tibshirani, R. Statistical significance for genomewide studies. Proc. Natl. Acad. Sci. U. S. A. 100, 16 (Aug. 2003), 9440-5.

[9] WeSt, M. Bayesian factor regression models in the large $\mathrm{p}$, small $\mathrm{n}$ paradigm. In Bayesian Stat. 7 - Proc. Seventh Val. Int. Meet. (2003), J. M. Bernardo, A. P. Dawid, J. O. Berger, M. West, D. Heckerman, M. Bayarri, and A. F. Smith, Eds., Oxford University Press, pp. 723-732.

[10] XU, J.-Z., AND WONG, C.-W. Hunting for robust gene signature from cancer profiling data: sources of variability, different interpretations, and recent methodological developments. Cancer Lett. 296, 1 (Oct. 2010), 9-16. 